\title{
Oxidants Spontaneously Released by Alveolar Macrophages of Cigarette Smokers Can Inactivate the Active Site of $\alpha 1$-Antitrypsin, Rendering It Ineffective as an Inhibitor of Neutrophil Elastase
}

\author{
Richard C. Hubbard, Fumitaka Ogushi, Gerald A. Fells, André M. Cantin," Sophie Jallat, \\ Michael Courtney, and Ronald G. Crystal \\ Pulmonary Branch, National Heart, Lung, and Blood Institute, National Institutes of Health, Bethesda, \\ Maryland 20892; and *Transgene SA, Strasbourg, 67000 France
}

\begin{abstract}
Current concepts relating to the pathogenesis of emphysema associated with cigarette smoking is that an imbalance exists within the lower respiratory tract between neutrophil elastase and the local anti-neutrophil elastase screen, enabling uninhibited neutrophil elastase to destroy the alveolar structures over time. The possible role of alveolar macrophages in contributing to this imbalance was investigated by evaluating the ability of cigarette smokers' alveolar macrophages to inactivate alpha 1 -antitrypsin ( $\alpha 1 \mathrm{AT})$, the major anti-neutrophil elastase of the human lower respiratory tract. In vitro, alveolar macrophages of smokers spontaneously released 2.5 -fold more superoxide anion and eightfold more $\mathrm{H}_{2} \mathrm{O}_{2}$ than macrophages of nonsmokers $(P<0.01$, both comparisons). Using a model system that reproduced the relative amounts of alveolar macrophages and $\alpha 1 \mathrm{AT}$ found in the epithelial lining fluid of the lower respiratory tract, we observed that smokers' macrophages caused a $60 \pm 5 \%$ reduction in the ability of $\alpha 1 \mathrm{AT}$ to inhibit neutrophil elastase. In marked contrast, under the same conditions, nonsmokers' macrophages had no effect upon the anti-neutrophil elastase function of $\alpha 1 \mathrm{AT}$. Addition of superoxide dismutase, catalase, mannitol, and methionine prevented inactivation of $\alpha 1 \mathrm{AT}$ by smokers' macrophages, implying that the release of oxidants mediated the inactivation of $\alpha 1 \mathrm{AT}$. In addition, by utilizing a recombinant DNA produced modified form of $\alpha 1 \mathrm{AT}$ containing an active site substitution (met $t^{358} \rightarrow$ val), the inactivation of $\alpha 1 \mathrm{AT}$ by smokers' alveolar macrophages was prevented, suggesting that the smokers' macrophages inactivate $\alpha 1 \mathrm{AT}$ by oxidizing the active site of the $\alpha 1 \mathrm{AT}$ molecule. These results suggest that in cigarette smokers, the alveolar macrophage can modulate the activity of $\alpha 1 \mathrm{AT}$ as an inhibitor of neutrophil elastase and thus play a role in the pathogenesis of emphysema associated with cigarette smoking.
\end{abstract}

\section{Introduction}

Alpha 1-antitrypsin ( $\alpha 1 \mathrm{AT}),{ }^{1}$ is a $52-\mathrm{kD}$ glycoprotein that serves as the major inhibitor of neutrophil elastase, a potent

Address reprint requests to Richard C. Hubbard, M.D., Pulmonary Branch, Building 10, Room 6D03, National Institutes of Health, Bethesda, MD 20892.

Dr. Cantin's present address is Hospitalier Universitaire de Sherbrooke, Unite de Recherche, Pulmonaire, Piece 3601, Sherbrooke, P.Q. Canada J1H 5 N4.

Received for publication 20 March 1987 and in revised form 30 June 1987.

1. Abbreviations used in this paper: $\alpha 1 \mathrm{AT}$, alpha 1-antitrypsin; ELF, epithelial lining fluid; MeO-SAAPV-NA, methoxy-succinyl-alanyl-

The Journal of Clinical Investigation, Inc.

Volume 80, November 1987, 1289-1295 serine protease that is capable of attacking most protein components of the extracellular matrix (1-3). The critical importance of $\alpha 1 \mathrm{AT}$ as an inhibitor of neutrophil elastase is highlighted by $\alpha$ IAT deficiency, a hereditary disorder associated with plasma $\alpha 1 \mathrm{AT}$ levels $<35 \%$ of normal and the development of emphysema in the third to fourth decades (4-7). In this context, the pathogenesis of the emphysema associated with $\alpha$ 1AT deficiency is conceptualized as an imbalance between $\alpha 1 \mathrm{AT}$ and neutrophil elastase in the alveolar structures such that there is insufficient $\alpha 1 \mathrm{AT}$ to provide a protective screen against the burden of neutrophils, and hence neutrophil elastase, in the local milieu $(4,8,9)$.

The same concepts have been extended to conceptualize the pathogenesis of the emphysema associated with cigarette smoking (10-15). Despite the fact that most of these individuals have normal levels of $\alpha 1 \mathrm{AT}$ in blood and lung $(16,17)$, the knowledge that $\alpha 1 \mathrm{AT}$ can be rendered impotent by oxidation has led to the concept that cigarette smoking may lead to an inactivation of $\alpha 1 \mathrm{AT}$ in the lung, leaving the lower respiratory tract without its normal anti-neutrophil elastase defenses $(1,12-15,18)$. This concept is supported by in vitro studies showing that cigarette smoke will inactivate $\alpha$ 1AT by oxidative mechanisms $(12,19-21)$ and that the $\alpha 1 \mathrm{AT}$ recovered from the epithelial surface of the lower respiratory tract of cigarette smokers has a reduced association rate constant for neutrophil elastase (22). Furthermore, the $\alpha 1 \mathrm{AT}$ recovered from the lung of cigarette smokers has been observed to have a decreased ability to inhibit porcine pancreatic elastase compared with the lung $\alpha$ lAT of nonsmokers $(13,23,24)$, although this observation has been disputed $(25,26)$.

Although it is compelling to invoke cigarette smoke, with its high concentration of oxidants (20), as a source of the oxidant burden that potentially could inactivate $\alpha 1 \mathrm{AT}$ in the lower respiratory tract, another potential source of oxidants in the lower respiratory tract of cigarette smokers are alveolar macrophages. In this regard: $(a)$ when activated, alveolar macrophages are capable of releasing oxidants, including superoxide anion, hydrogen peroxide, and hydroxyl radical $(27,28)$; (b) the number of alveolar macrophages in the lower respiratory tract of cigarette smokers is increased severalfold $(17,29$, $30)$; (c) when activated in vitro, alveolar macrophages can release sufficient oxidants to render $\alpha 1 \mathrm{AT}$ an ineffective inhibitor of neutrophil elastase $(31,32)$; and $(d)$ alveolar macrophages recovered from the lungs of cigarette smokers are spontaneously releasing oxidants $(29,33)$. In the context of these observations it is reasonable to hypothesize that alveolar macrophages in the lower respiratory tract of cigarette smokers

alanyl-prolyl-valine nitroanilide; $\mathrm{O}_{2}^{\dot{\prime}}$, superoxide anion; SOD, superoxide dismutase. 
may be spontaneously releasing sufficient oxidants to inactivate $\alpha 1 \mathrm{AT}$ in the local milieu.

To evaluate this hypothesis, we recovered alveolar macrophages from the lower respiratory tract of nonsmokers and smokers, and evaluated the ability of these cells to modulate the activity of $\alpha 1 \mathrm{AT}$ as an inhibitor of neutrophil elastase. In addition, by utilizing a recombinant DNA-produced modified form of $\alpha 1$ AT containing an active site substitution $\left(\right.$ met $^{358} \rightarrow$ val) rendering it resistant to oxidation but still active as an inhibitor of neutrophil elastase, we have demonstrated that the spontaneous inactivation of $\alpha 1 \mathrm{AT}$ by macrophages of cigarette smokers occurs, at least in part, by oxidation of the active site of the $\alpha 1 \mathrm{AT}$.

\section{Methods}

Study population. The study population consisted of 5 healthy nonsmoking individuals (three male, two female; age $31 \pm 7 \mathrm{yr}$ ) and 10 healthy smoking individuals (six male, four female; age $32 \pm 6 \mathrm{yr}$ ) with an average cigarette smoking history of $27 \pm 11$ pack years. (Data is presented as mean $\pm S E$ of the mean; all statistical comparisons were carried out using the two-tailed Student's $t$ test.) All individuals were free of lung disease as determined by the combined criteria of having normal histories, physical examinations, chest $\mathrm{x}$ rays, and lung function tests (34).

Lower respiratory tract inflammatory cells. Inflammatory cells were recovered from the lower respiratory tract of nonsmokers and smokers by bronchoalveolar lavage as previously described (35). Alveolar macrophages were purified by adherence $\left(1 \mathrm{~h}, 37^{\circ}\right)$ at $5 \times 10^{5}$ cells in $1 \mathrm{ml}$ in 24-well tissue culture plates (Falcon Labware, Oxnard, CA) in RPMI medium (Gibco, Grand Island, NY) containing $10 \%$ calf serum (Whitaker M. A. Bioproducts, Walkersville, MD). In all cases the alveolar macrophages were $>95 \%$ pure and $>90 \%$ viable (as assessed by trypan blue). The volume of epithelial lining fluid (ELF) recovered, quantified using the urea method (36), was $2.0 \pm 1.0 \mathrm{ml}$ for nonsmokers and $2.7 \pm 1.1 \mathrm{ml}$ for smokers $(P>0.2)$. Concentrations of $\alpha 1 \mathrm{AT}$ in ELF were quantified using an enzyme-linked immunoassay (in quadruplicate) with a standard $\alpha 1 \mathrm{AT}$ that had been quantified by amino acid analysis (37).

The spontaneous release of superoxide anion $\left(\mathrm{O}_{2}^{\dot{*}}\right)$ and $\mathrm{H}_{2} \mathrm{O}_{2}$ by the purified alveolar macrophages of the nonsmokers and smokers was quantified in vitro $(38,39)$. Briefly, for $\mathrm{O}_{2}^{-}$, the alveolar macrophages $\left(5 \times 10^{5} /\right.$ well $)$ in 24-well plates were incubated in $0.5 \mathrm{ml}$ of Hanks' balanced salt solution (HBSS; Gibco) containing $80 \mathrm{~nm}$ ferricytochrome $c$ (Type III, Sigma Chemical Co., St. Louis, MO). After incubation for $30 \mathrm{~min}$ at $37^{\circ}$, the amount of $\mathrm{O}_{2}^{-}$released into the medium was quantified at $550 \mathrm{~nm}$. For the measurement of the spontaneous release of $\mathrm{H}_{2} \mathrm{O}_{2}$, HBSS containing $0.28 \mathrm{mM}$ phenol red, 8.5 $\mathrm{U} / \mathrm{ml}$ horseradish peroxidase (Type II, Sigma Chemical Co.) was added and the macrophages incubated for $30 \mathrm{~min}$ at $37^{\circ}$. The amount of $\mathrm{H}_{2} \mathrm{O}_{2}$ in the supernatant was then determined by the addition of $10 \mu \mathrm{l}$ of $1 \mathrm{~N} \mathrm{NaOH}$ and the absorbance read at $610 \mathrm{~nm}$. The concentration of $\mathrm{H}_{2} \mathrm{O}_{2}$ was based on a standard curve using various concentrations of reagent grade $\mathrm{H}_{2} \mathrm{O}_{2}$.

Source of $\alpha 1 A T$. Normal human $\alpha 1 \mathrm{AT}$ was purified from the plasma of an individual homozygous for the $\mathrm{M} 1\left(\mathrm{val}^{213}\right)$ form of $\alpha 1 \mathrm{AT}$ as identified by the combined criteria of isoelectric focusing, serum $\alpha 1 \mathrm{AT}$ levels, family studies, and BstEII restriction endonuclease mapping of genomic DNA $(40,41)$. Purification of $\alpha 1 \mathrm{AT}$ from plasma was accomplished by positive selection affinity chromatography (42). The purified $\alpha 1 \mathrm{AT}$ was concentrated by pressure filtration (YM-10 membrane, Amicon Corp., Danvers, MA) and stored in aliquots in liquid nitrogen vapor until use. The final preparation was $>95 \%$ pure as assessed by sodium dodecyl sulfate-polyacrylamide gel electrophoresis.

Recombinant $\alpha 1 \mathrm{AT}$ was produced in Escherichia coli as previously described $(43,44)$, using an $\alpha 1$ AT complementary DNA (cDNA) obtained from translation of human liver RNA. Competent TGE900 $E$. coli cells were transformed with an expression plasmid containing the $\alpha 1 \mathrm{AT}$ cDNA and the recombinant $\alpha 1 \mathrm{AT}$ purified from bacterial lysates. Two forms of recombinant $\alpha 1 \mathrm{AT}$ were produced. The normal form ( $\mathrm{met}^{358} \mathrm{r} \alpha \mathrm{lAT}$ ) has the identical sequence of the $\mathrm{Ml}\left(\mathrm{val}^{213}\right)$ form of human $\alpha 1 \mathrm{AT}$ present in plasma except for an additional $\mathrm{NH}_{2}$-terminal methionine and the absence of carbohydrates. It is designated $\mathrm{met}^{358} \mathrm{r} \alpha \mathrm{AT}$ to indicate that it contains a methionine in the active inhibitory site, as does the normal $\mathrm{M} 1\left(\mathrm{val}^{213}\right)$ protein. The val ${ }^{358}$ r $\alpha 1$ AT form was identical to the met ${ }^{358}$ r $\alpha 1$ AT except for the substitution of $\mathrm{val}^{358}$ at the active site. The val ${ }^{358}$ r $\alpha 1 \mathrm{AT}$ has been shown to be similar to the met ${ }^{358} \mathrm{r} \alpha 1 \mathrm{AT}$ in terms of its ability to inhibit human neutrophil elastase, but the val ${ }^{358} \mathrm{r} \alpha 1 \mathrm{AT}$ is much more resistant to oxidation (44-48).

Evaluation of ability of the $\alpha 1 A T$ preparations to inhibit neutrophil elastase. The ability of $\alpha 1 \mathrm{AT}$ to inhibit neutrophil elastase was evaluated using two assays: $(a)$ the time-dependent inhibition of equimolar concentration of neutrophil elastase and $\alpha 1 \mathrm{AT}$; and $(b)$ the time-independent inhibition of neutrophil elastase by increasing concentrations of $\alpha 1 \mathrm{AT}$. Both assays were carried out using a neutrophil elastase standard for which the activity had been determined (37).

The time-dependent inhibition of purified $\alpha 1 \mathrm{AT}$ with neutrophil elastase was carried out by the method of Beatty et al. (11) with minor modifications described by Straus et al. (37). In brief, the $\alpha 1 \mathrm{AT}$ was titrated using neutrophil elastase to determine the percentage of active $\alpha 1 \mathrm{AT}$ in the preparation. Equimolar amounts of elastase and active $\alpha 1 \mathrm{AT}$ ( $1 \mathrm{nM}$ each) were then reacted at $23^{\circ}$ in a $1-\mathrm{ml}$ reaction volume containing $100 \mathrm{mM}$ Hepes, $\mathrm{pH} 7.5,0.5 \mathrm{M} \mathrm{NaCl}$, and $0.1 \%$ Brij-35. Residual elastase activity at various times was determined by terminating the reaction by the addition of the specific neutrophil elastase substrate methoxy-succinyl-alanyl-alanyl-prolyl-valine nitroanilide (MeO-SAAPV-NA; $1 \mathrm{mM}$; Sigma Chemical Co.) and the residual elastase activity measured at $410 \mathrm{~nm}$ as a change in optical density per minute using a DU-7 spectrophotometer (Beckman Instruments, Fullerton, CA). At each time point the percent inhibition of elastase was then quantified.

Time-independent inhibition of neutrophil elastase by $\alpha 1 \mathrm{AT}$ was determined by incubating a fixed amount $(2 \mathrm{nM})$ of active neutrophil elastase with various concentrations $(0-8 \mathrm{nM})$ of $\alpha 1 \mathrm{AT}$ at $23^{\circ}$ for $2 \mathrm{~h}$, a time at which the time-dependent assays demonstrated were at least twofold longer than necessary for reaction of neutrophil elastase with the $\alpha 1 \mathrm{AT}(11,49)$. After the incubation, the neutrophil elastase-specific substrate MeO-SAAPV-NA was added and percent residual elastase activity was determined as previously described.

Evaluation of ability of smoker alveolar macrophages to spontaneously inactivate $\alpha 1 A T$ as an inhibitor of elastase. The ability of smokers' alveolar macrophages to spontaneously inactivate $\alpha 1 \mathrm{AT}$ was evaluated using an in vitro assay that mimicked the numbers of macrophages and concentrations of $\alpha 1 \mathrm{AT}$ in the epithelial lining fluid of the lower respiratory tract. To accomplish this, alveolar macrophages recovered from the lower respiratory tract of smokers and nonsmokers were exposed in vitro to purified plasma $\alpha 1 \mathrm{AT}$ (type M1[val ${ }^{213}$ ], see previous section) in a chamber designed to permit only low-molecular weight products of the macrophages to reach the $\alpha 1 \mathrm{AT}$. The chamber was divided in two sections, a lower $\alpha 1 \mathrm{AT}$-containing section and an upper macrophage chamber. The total volume of the chamber was $1,400 \mu \mathrm{l}$ (upper section, 1,000 $\mu \mathrm{l}+$ lower section, $400 \mu \mathrm{l}$ ). Separating the chambers was a dialysis membrane (Union Carbide Corp., Danbury, CT) with a molecular weight cutoff of $12,000-14,000 \mathrm{kD}$. Alveolar macrophages $\left(2 \times 10^{6}\right)$ from a nonsmoker or a smoker were placed in HBSS containing $0.1 \%$ glucose in the upper chamber and $\alpha 1 \mathrm{AT}$ ( $1 \mu \mathrm{M}$ in HBSS containing $0.1 \%$ glucose) was placed in the lower chamber. The numbers of alveolar macrophages and the amounts of $\alpha 1 \mathrm{AT}$ used were always in the ratio of $4.65 \times 10^{9}$ macrophages $/ \mu \mathrm{mol}$ $\alpha 1 \mathrm{AT}$. This value was based on the average ratio of macrophages to $\alpha 1 \mathrm{AT}$ recovered in the ELF of nonsmokers, and is approximately threefold less than that observed in smokers, i.e., the design was based 
on that found in normals and was biased against the increased ratio of macrophages to $\alpha 1 \mathrm{AT}$ present in the lower respiratory tract of smokers. Control samples contained $\alpha 1 \mathrm{AT}$ in the lower chamber but no added alveolar macrophages. After the chambers were incubated for $18 \mathrm{~h}$ at $37^{\circ}, \alpha 1 \mathrm{AT}$ was retrieved from the lower portion of the chamber and then evaluated for its ability to inhibit neutrophil elastase, as previously described.

To demonstrate that low-molecular weight mediators of smoker alveolar macrophages that inactivated the $\alpha 1 \mathrm{AT}$ were oxidants, parallel incubations were carried out using alveolar macrophages from smokers and purified plasma $\alpha 1 \mathrm{AT}$ but with the addition of antioxidants to the chambers. Superoxide dismutase (SOD; $400 \mathrm{U} / \mathrm{ml}$, Type III, Sigma Chemical Co.), catalase $(1,000 \mathrm{U} / \mathrm{ml}$, bovine liver, Sigma Chemical Co.), or a combination of both antioxidants were added to both the macrophage and $\alpha 1 \mathrm{AT}$ portions of the chambers and the chambers incubated as previously described. In selected experiments the hydroxyl radical $\left(\mathrm{OH}^{\circ}\right)$ scavenger mannitol $(1 \mathrm{mM})$ or methionine (1 mM) (which inactivates $\mathrm{HOCl}$ ) was added. After incubation, the $\alpha 1 \mathrm{AT}$ was retrieved and its ability to inhibit porcine pancreatic elastase (Calbiochem-Behring Corp., La Jolla, CA) was measured. Pancreatic elastase was specifically used in this experiment because when the active site met ${ }^{358}$ residue of $\alpha 1 \mathrm{AT}$ is oxidized, the $\alpha 1 \mathrm{AT}$ molecule completely loses its ability to inhibit porcine pancreatic elastase. (In contrast, it is still able to inhibit neutrophil elastase, albeit with a markedly reduced association rate constant [11]). Thus, although porcine pancreatic elastase is not the normal physiologic target of human $\alpha 1 \mathrm{AT}$ in vivo, it offers a convenient method to analyze the protective effect of antioxidants. The reaction between the $\alpha 1 \mathrm{AT}$ recovered from the chambers and the porcine pancreatic elastase was carried out in a $1-\mathrm{ml}$ reaction volume containing $100 \mathrm{mM}$ Tris- $\mathrm{HCl}, \mathrm{pH} 8.3,0.5 \mathrm{M}$ $\mathrm{NaCl}, 0.1 \%$ Brij-35, and $8 \mathrm{nM}$ porcine pancreatic elastase (11). Varying concentrations of $\alpha 1 \mathrm{AT}$ were added and the reaction mixture incubated for $3 \mathrm{~h}$ at $23^{\circ}$. After incubation, the porcine pancreatic elastasespecific substrate ( $n$-succinyl-alanyl-alanyl-alanyl-NA, $1 \mathrm{mM}$, Calbiochem-Behring Corp.) was added to the incubation. The residual pancreatic elastase activity was then quantified by measuring the change in optical density at $410 \mathrm{~nm}$, and percent inhibition of elastase was calculated as previously described.

To directly demonstrate the involvement of the $\alpha 1 \mathrm{AT}$ met $^{358}$ residue as the target for the smoker alveolar macrophage-released oxidants as the mechanism for inactivating the $\alpha 1 \mathrm{AT}$, we carried out identical experiments as described for the smoker macrophages and the plasma $\alpha 1 \mathrm{AT}$, but recombinant forms of $\alpha 1 \mathrm{AT}$, met ${ }^{358} \mathrm{r} \alpha 1 \mathrm{AT}$, and val $^{358}$ r $\alpha 1$ AT were substituted for the plasma $\alpha 1$ AT. Briefly, smokers' alveolar macrophages were added to the upper portion of a chamber across a dialysis membrane from either met ${ }^{358} \alpha 1 \mathrm{AT}$ or val ${ }^{358} \alpha 1 \mathrm{AT}$ in the same ratios used previously. After incubation for $18 \mathrm{~h}$ at $37^{\circ}$ the recombinant $\alpha 1 \mathrm{AT}$ was retrieved and evaluated for its ability to inhibit neutrophil elastase as previously described.

\section{Results}

Relationship between alveolar macrophages and $\alpha 1 A T$ on the alveolar epithelial surface. Evaluation of lavage fluid demonstrated that, relative to the amounts of $\alpha 1$ AT present, the environment of the epithelial lining fluid of the lower respiratory tract of nonsmokers and cigarette smokers were substantially different both in number of alveolar macrophages and amount of oxidants released per hour. In this regard, nonsmokers had an average of $21.4 \pm 14.2 \times 10^{3}$ alveolar macrophages per $\mu l$ of lower respiratory tract epithelial lining fluid, whereas the smokers had $43.2 \pm 26.3 \times 10^{3} / \mu$ l ELF $(P<0.01)$. However, the concentration of $\alpha 1 \mathrm{AT}$ in ELF was similar in the two groups (nonsmokers, $4.5 \pm 0.8 \mu \mathrm{M}$; smokers, $3.21 \pm 1.0 \mu \mathrm{M} ; P$ $>0.1$ ). The ELF of smokers contained nearly threefold more alveolar macrophages per mole of $\alpha 1 \mathrm{AT}$ than did nonsmokers
(Fig. $1 A ; P<0.01$ ). Not only did the smokers have relatively more alveolar macrophages, but the macrophages were activated and spontaneously releasing more oxidants than the macrophages of nonsmokers. In this context nonsmoker alveolar macrophages were releasing $18.2 \pm 8.3 \mathrm{nmol} \mathrm{O}_{2}^{-} / 10^{6} \mathrm{al}-$ veolar macrophages per $\mathrm{h}$ and $1.6 \pm 0.6 \mathrm{nmol} \mathrm{H}_{2} \mathrm{O}_{2} / 10^{6}$ macrophages per $\mathrm{h}$, whereas smoker macrophages were releasing $42.4 \pm 13.3 \mathrm{nmol} \mathrm{O}_{2}^{-} / 10^{6}$ macrophages per $\mathrm{h}$ and $13.1 \pm 4.2$ nmol $\mathrm{H}_{2} \mathrm{O}_{2} / 10^{6}$ macrophages per $\mathrm{h}$ (nonsmokers vs. smokers, $P<0.01$ for $\mathrm{O}_{2}^{-}$and $\mathrm{H}_{2} \mathrm{O}_{2}$, respectively). When considered in terms of amounts of $\alpha 1 \mathrm{AT}$ present in ELF, it was apparent that smokers' alveolar macrophages present a burden of oxidants markedly greater than do nonsmoker macrophages. For example, nonsmoker macrophages spontaneously released $84 \pm 12$ $\mathrm{mol} / \mathrm{h}^{-1} \mathrm{O}_{2}^{+}$per mol $\alpha 1 \mathrm{AT}$ in ELF, whereas smoker macrophages released $570 \pm 120 \mathrm{~mol} / \mathrm{h}^{-1} \mathrm{O}_{2}^{\prime}$ per mol $\alpha 1 \mathrm{AT}(P$ $<0.005$ ). The burden of $\mathrm{H}_{2} \mathrm{O}_{2}$ released by the alveolar macrophages was even greater. Smoker alveolar macrophages released 28-fold more $\mathrm{H}_{2} \mathrm{O}_{2}$ per mol of $\alpha 1 \mathrm{AT}$ in ELF than did nonsmoker alveolar macrophages (Fig. $1 B, P<0.001$ ). Thus, on the average, the oxidant burden from alveolar macrophages faced by a molecule of $\alpha$ 1 AT within the smokers' alveolus is far greater than that within the nonsmokers' alveolus, and consequently the likelihood of oxidative alteration of the properties of the $\alpha$ 1AT molecule is correspondingly much greater within the smokers' lung than in the lung of the nonsmoker.

Analysis of ability of nonsmoker and smoker alveolar macrophages to inactivate $\alpha 1 A T$ as an inhibitor of neutrophil elastase. When alveolar macrophages were incubated with $\alpha 1 \mathrm{AT}$ in the in vitro system designed to reproduce the microenvironment of ELF, very different effects were observed between smokers' and nonsmokers' alveolar macrophages on the antielastase function of the $\alpha 1 \mathrm{AT}$ present. The nonsmokers' macrophages caused almost no loss of function of the $\alpha 1 \mathrm{AT}$ as an inhibitor of neutrophil elastase (Fig. 2). In contrast, smoker alveolar macrophages caused a significant loss of the antielastase function of $\alpha 1 \mathrm{AT}$. The loss of antielastase function was present at all elastase- $\alpha 1 \mathrm{AT}$ incubation intervals measured. Lack in increase in antielastase function with time suggests that a significant proportion of the population of $\alpha 1$ AT molecules were rendered completely impotent as inhibitors of neutrophil elastase. Strikingly, the ability of smokers' macro-
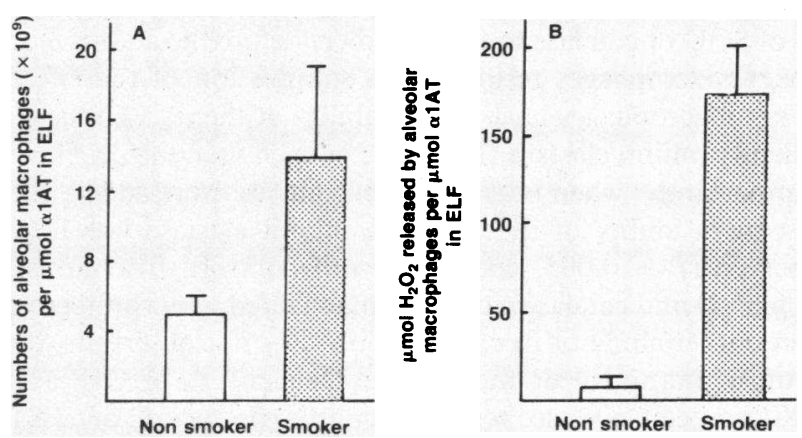

Figure 1. Relationship of alveolar macrophages and $\alpha 1 \mathrm{AT}$ in the epithelial lining fluid of the lower respiratory tract of nonsmokers and smokers. $(A)$ Ratio of number of alveolar macrophages recovered by lavage to amount of $\alpha 1 \mathrm{AT}$ in the same lavage sample. $(B)$ Ratio of amount of $\mathrm{H}_{2} \mathrm{O}_{2}$ spontaneously released by alveolar macrophages/ $\mathrm{h}^{-1}$ to the amount of $\alpha 1 \mathrm{AT}$ in ELF. 


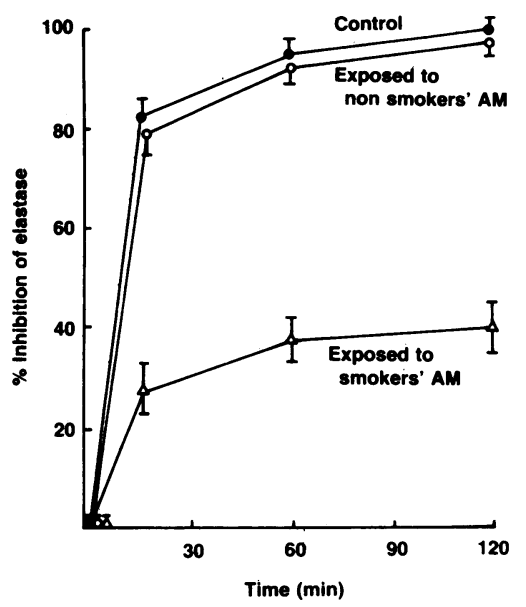

Figure 2. Effect of lowmolecular weight mediators spontaneously released by alveolar macrophages of nonsmokers and smokers to suppress the ability of $\alpha 1$ AT to inhibit neutrophil elastase. $\alpha 1 \mathrm{AT}$ ( 1 $\mu \mathrm{mol}$ ) was exposed to alveolar macrophages $\left(10^{6} / \mathrm{ml} ; 4.65 \times 10^{9}\right.$ macrophages $/ \mu \mathrm{mol}$ $\alpha 1 \mathrm{AT})$ in chambers for $18 \mathrm{~h}, 37^{\circ}$. Dialysis tubing (mol wt cutoff, 12-14 kD) separated the macrophages from

the $\alpha 1 \mathrm{AT}$. The $\alpha 1 \mathrm{AT}$ was recovered from the chamber and evaluated for its ability to inhibit neutrophil elastase over time ( $2 \mathrm{nM}$ elastase, 0-8 nM $\alpha 1 \mathrm{AT}$ ). Shown is the elastase inhibitory capacity of $\alpha 1 \mathrm{AT}$ incubated in the chamber without alveolar macrophages on the other side of the membrane ( $\bullet)$; $\alpha 1$ AT exposed to alveolar macrophages of nonsmokers (O); and $\alpha 1 \mathrm{AT}$ exposed to alveolar macrophages of smokers $(\Delta)$.

phages to inactivate $\alpha 1 \mathrm{AT}$ occurred despite that fact that conditions within the assay mimicked those within the nonsmokers' alveolus rather than those of the smoker alveolus. In this regard, when the number of smokers' alveolar macrophages was increased beyond the ratio of alveolar macrophages to $\alpha 1 \mathrm{AT}$ ELF found in nonsmokers, the loss of antineutrophil activity was even greater, with the loss of activity proportional to numbers of macrophages in the chamber (data not shown). When put in the context of the microenvironment of the ELF of the smoker, where the ratio of macrophages per micromole $\alpha$ IAT ELF is threefold greater than that used in this study, the observed effect upon $\alpha 1 \mathrm{AT}$ (Fig. 2) probably represents the minimum reduction of $\alpha 1 \mathrm{AT}$ antielastase activity ongoing in the lower respiratory tract of smokers.

Effect of antioxidants on ability of mediators released by smoker alveolar macrophages to interfere with $\alpha 1$ AT antielastase function. When antioxidants were added to the incubations containing smoker alveolar macrophages and $\alpha 1 \mathrm{AT}$, the suppression of $\alpha 1 \mathrm{AT}$ antielastase function caused by the smoker macrophages was not observed. In this regard, addition of SOD or catalase to the chambers before the addition of smoker macrophages resulted in a suppression of the effect smoker macrophages have on the ability of the $\alpha 1 \mathrm{AT}$ to subsequently inhibit elastase $(P<0.01$ for each vs. control; Table I). Importantly, when both SOD and catalase were added, the subsequent ability of the $\alpha 1 \mathrm{AT}$ to inhibit elastase was fully preserved $(P<0.005$, compared with control). In contrast, when SOD and catalase were heat-inactivated, their protective effect (either alone or in combination) was not observed. Addition of mannitol or methionine (scavengers of $\mathrm{OH}^{*}$ and $\mathrm{HOCl}$, respectively) also resulted in partial protection of $\alpha 1 \mathrm{AT}$ antielastase capacity (mannitol, $64+4, P<0.05$ vs. control; methionine, $63+7, P<0.05$ vs. control). These findings indicate that low-molecular weight oxidants were the mediators responsible for the observed loss of the elastase inhibitory function of $\alpha 1 \mathrm{AT}$ after its incubation with smokers' alveolar macrophages. This is consistent with the observation that
Table I. Effect of Antioxidants on Ability of Low-Molecular Weight Mediators Spontaneously Released by Alveolar Macrophages of Cigarette Smokers to Suppress the Ability of $\alpha 1$-Antitrypsin to Inhibit Elastase

\begin{tabular}{ll}
\hline Condition & $\begin{array}{l}\text { Inhibition } \\
\text { of Elastase }^{*}\end{array}$ \\
\hline & $\%$ \\
$\alpha 1 \mathrm{AT}$ exposed to smoker macrophages & $27 \pm 12$ \\
+ in the presence of SOD & $68 \pm 9$ \\
+ in the presence of catalase & $71 \pm 2$ \\
+ in the presence of SOD + catalase $^{\S}$ & $98 \pm 2$ \\
+ in the presence of mannitol & $64 \pm 4$ \\
+ in the presence of methionine & $63 \pm 7$
\end{tabular}

* All assays contained $8 \mathrm{nM}$ active porcine pancreatic elastase and 0-20 nM $\alpha 1 \mathrm{AT} ; 100 \%$ inhibition = all of the elastase was inhibited. $\$ 400 \mathrm{U} / \mathrm{ml}$. Heat-inactivated SOD gave no protection $(26 \pm 11 \%$ inhibition of elastase). ${ }^{\S} 1,000 \mathrm{U} / \mathrm{ml}$. Heat-inactivated catalase gave no protection ( $30 \pm 15 \%$ inhibition of elastase). "1 mM. "1 mM.

smoker macrophages release exaggerated amounts of $\mathrm{H}_{2} \mathrm{O}_{2}$, $\mathrm{O}_{2}^{\prime}$ and $\mathrm{OH}^{\circ}$, and can utilize myeloperoxidase (either their own endogenous enzyme or myeloperoxidase scavenged from neutrophils) to generate the strong oxidant $\mathrm{HOCl}$.

The met $^{358}$ active site of $\alpha 1 A T$ as a target for smoker macrophages. The ability to produce recombinant forms of $\alpha 1 \mathrm{AT}$ that are identical except for a single amino acid substitution at the active inhibitory site provides a means to confirm that at least part of the mechanism by which alveolar macrophages inactive $\alpha 1 \mathrm{AT}$ as an inhibitor of elastase is by oxidizing the met $^{358}$ residue at the site that is central to the elastase-inhibitory function of the $\alpha 1 \mathrm{AT}$ molecule. In this regard, cigarette smokers' alveolar macrophages caused a nearly total loss of the ability of met $^{358}$ r $\alpha 1$ AT to inhibit neutrophil elastase (Fig. $3 A$ ). In contrast, the ability of $\mathrm{val}^{358} \mathrm{r} \alpha \mathrm{AAT}$, to inhibit neutrophil elastase was not significantly affected by exposure to cigarette smokers' macrophages (Fig. $3 \mathrm{~B}$ ). On the average, when compared with control met ${ }^{358}$ r $\alpha 1 \mathrm{AT}$ not exposed to macrophages, the met ${ }^{358}$ r $\alpha 1 \mathrm{AT}$ exposed to smoker macrophages was only $14 \pm 6 \%$ as active (Fig. $3 C$ ). In contrast, val $^{358}$ r $\alpha 1$ AT retained $84 \pm 8 \%$ of its activity $(P<0.005)$. Because the only difference between met $^{358}$ r $\alpha 1 \mathrm{AT}$ and $\mathrm{val}^{358}$ r $\alpha$ lAT is the substitution of valine for methionine at the active site of the $\alpha 1 \mathrm{AT}$ molecule, this differential effect strongly suggests that the mechanism of suppression of $\alpha 1$ AT antielastase activity by smokers' macrophages is associated with the action of oxidants upon the active site methionine residue.

\section{Discussion}

The finding that cigarette smokers' alveolar macrophages spontaneously are releasing sufficient amounts of oxidants to modulate the neutrophil elastase-inhibitory function of $\alpha 1 \mathrm{AT}$ provides insight into the mechanisms through which cigarette smoking is linked to a markedly increased risk for the development of emphysema. In cigarette smokers, alveolar macrophages are present in the lower respiratory tract in numbers severalfold greater than in nonsmokers $(17,29,30)$. Characteristically they accumulate in terminal bronchioles, where they represent an early pathologic lesion in asymptomatic cig- 

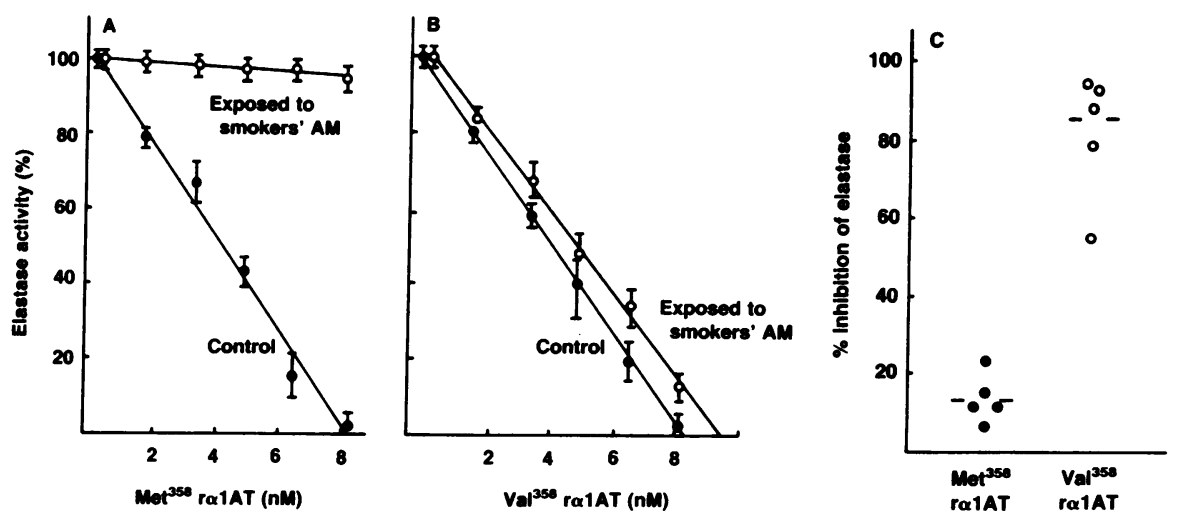

Figure 3. Effect of low-molecular weight mediators spontaneously released by alveolar macrophages of cigarette smokers on the ability of recombinant forms of $\alpha 1 \mathrm{AT}$ to inhibit neutrophil elastase. Recombinant $\alpha 1 \mathrm{AT}(1 \mu \mathrm{M})$ was separated from smokers' alveolar macrophages as in Fig. 2. Control $\alpha 1 \mathrm{AT}$ was placed in identical chambers but not exposed to alveolar macrophages. After $18 \mathrm{~h}, 37^{\circ}, \alpha 1 \mathrm{AT}$ was retrieved and tested for its ability to inhibit neutrophil elastase ( $8 \mathrm{nM})$ over a 1-h incubation period. $(A)$ Recombinant met ${ }^{358}$ $\alpha 1 \mathrm{AT}$. Shown is an example from one individual; data are presented as percent ac-

tivity of the neutrophil elastase remaining. (B) Recombinant $v^{358} \alpha 1$ AT. Shown are data from one individual presented as in panel $A$. (C) Comparison of proportion of recombinant met ${ }^{358} \alpha 1 \mathrm{AT}$ and recombinant val ${ }^{358} \alpha 1 \mathrm{AT}$ capable of inhibiting neutrophil elastase after exposure to low-molecular weight products spontaneously released by alveolar macrophages of cigarette smokers. Recombinant met ${ }^{358} \alpha 1 \mathrm{AT}$ and val ${ }^{358}$ $\alpha 1 \mathrm{AT}$ were exposed to smokers' alveolar macrophages in chambers as described in panels $A$ and $B$. The incubation was continued for $18 \mathrm{~h}$ at $37^{\circ}$, after which the elastase-inhibitory capacity of the recombinant $\alpha 1 \mathrm{AT}$ was determined. Each point represents a single individual.

arette smokers (50). Alveolar macrophages, by virtue of their increased numbers and exaggerated production of oxidants $(29,32,33)$, are ideal candidates to effect the functional integrity of the antielastase screen of the lower respiratory tract. In this regard, in the context of the present study, it is reasonable to conceptualize that macrophages create local areas within the alveolus in which there is deficient antineutrophil elastase protection due to oxidation of $\alpha 1 \mathrm{AT}$. In this scenario, lack of antielastase screen makes these areas of the lung vulnerable to elastase released by neutrophils or by macrophages that have ingested neutrophil elastase $(51,52)$. The result would be development of localized tissue destruction that ultimately may become the clinical disorder emphysema.

Oxidation of $\alpha 1 \mathrm{AT}$ by smokers' alveolar macrophages is also consistent with the study by Carp et al. (23) that observed oxidized $\alpha 1 \mathrm{AT}$ in bronchoalveolar lavage fluid obtained from smokers' lungs. These observations are consistent with the knowledge that oxidized $\alpha 1 \mathrm{AT}$ has a reduced association rate constant for neutrophil elastase (11) and that smoker lower respiratory tract $\alpha 1 \mathrm{AT}$ has a lower association rate constant for neutrophil elastase than does nonsmoker lower respiratory tract $\alpha 1 \mathrm{AT}$ (22). However, the demonstration that $\alpha 1 \mathrm{AT}$ can be oxidized by smokers' macrophages does not necessarily imply that a significant proportion of the $\alpha 1 \mathrm{AT}$ in all regions within the lower respiratory tract is oxidized. A more reasonable conceptualization based upon this model is that some $\alpha 1 \mathrm{AT}$ is inactivated by oxidants released by the macrophages in areas of macrophage accumulation. This process may not be occurring to the same degree everywhere within the lung, due to local differences in macrophage concentration, availability of antioxidants, transudation of plasma, or other factors that could have the effect of decreasing the local intensity of alveolar macrophage exposure to $\alpha 1 \mathrm{AT}$. Furthermore, because cigarette smoke itself can oxidize $\alpha 1 \mathrm{AT}$, the extent, type, and technique of smoking may also influence the status of $\alpha 1 \mathrm{AT}$ in different areas of the lower respiratory tract $(19,24)$. Hence, $\alpha 1 \mathrm{AT}$ recovered by bronchoalveolar lavage may contain active and inactive $\alpha 1 \mathrm{AT}$ in varying proportions. This heterogeneity may explain, at least in part, why some studies have shown that the population of $\alpha 1 \mathrm{AT}$ molecules recovered from the lower respiratory tract of cigarette smokers is relatively unable to inhibit pancreatic elastase compared with that recovered from nonsmokers $(13,23,24)$, whereas other studies have not shown such a difference $(25,26)$.

The fact that alveolar macrophages in the lower respiratory tract of cigarette smokers are capable of modifying $\alpha 1 \mathrm{AT}$ in the local milieu by oxidant mechanisms has implications for future approaches to investigating therapeutic strategies for preventing emphysema associated with cigarette smoking. First, because alveolar macrophages are replaced slowly (53, 54), this may explain why some individuals continue to develop lung destruction even after discontinuing cigarette smoking. Therefore, therapeutic modalities directed at suppressing the exaggerated oxidant release by the macrophages may be useful. Second, antioxidants may provide an important means of preserving the antielastase screen of the lower respiratory tract. Finally, while there are theoretical dangers in providing a "super" form of $\alpha 1$ AT that cannot be inactivated, the $\mathrm{val}^{358}$ form of recombinant $\alpha 1 \mathrm{AT}$ may be one means to protect the lower respiratory tract against a burden of neutrophil elastase when there is a concomitant burden of oxidants such as those released by alveolar macrophages of cigarette smokers.

\section{References}

1. Travis, J., and G. Salvesen. 1983. Human plasma proteinase inhibitors. Ann. Rev. Biochem. 52:655-709.

2. Janoff, A., R. White, H. Carp, S. Harel, R. Dearing, and D. Lee. 1979. Lung injury induced by leukocytic proteases. Am. J. Pathol. 97:111-129.

3. Bieth, J. G. 1986. Elastases: catalytic and biological properties. In Regulation of Matrix Accumulation. R. Mechan, editor. Academic Press, New York. 217-320.

4. Gadek, J. E., and R. G. Crystal. 1982. $\alpha 1$-Antitrypsin deficiency. In The Metabolic Basis of Inherited Disease. J. B. Stanbury, J. B. Wyngaarden, D. S. Fredrickson, J. L. Goldstein, and M. S. Brown, editors. McGraw-Hill Book Co., New York. 1450-1467.

5. Carrell, R. W., J.-O. Jeppsson, C.-B. Laurell, S. O. Brennan, M. C. Owen, L. Vaughan, and D. R. Boswell. 1982. Structure and variation of human $\alpha 1$-antitrypsin. Nature (Lond.). 298:329-334.

6. Kueppers, F., and L. F. Black. 1974. $\alpha 1$-Antitrypsin and its deficiency. Am. Rev. Respir. Dis. 110:176-194. 
7. Morse, J. O. 1978. Alpha 1-antitrypsin deficiency. N. Engl. J. Med. 299:1045-1048, 1099-1105.

8. Gadek, J. E., G. A. Fells, R. L. Zimmerman, S. I. Rennard, and R. G. Crystal. 1981. Antielastases of the human alveolar structures. Implications for the protease-antiprotease theory of emphysema. $J$. Clin. Invest. 68:889-898.

9. Janoff, A. 1985. Elastases and emphysema. Current assessment of the protease-antiprotease hypothesis. Am. Rev. Respir. Dis. 132:417-433.

10. Johnson, D., and J. Travis. 1979. The oxidative inactivation of human $\alpha$-1-proteinase inhibitor: further evidence of methionine at the reactive center. J. Biol. Chem. 254:4022-4026.

11. Beatty, K., J. Bieth, and J. Travis. 1980. Kinetics of association of serine proteinases with native and oxidized $\alpha$-1-proteinase inhibitor and $\alpha$-1-antichymotrypsin. J. Biol. Chem. 255:3931-3934.

12. Janoff, A., and H. Carp. 1977. Possible mechanisms of emphysema in smokers: cigarette smoke condensate suppresses protease inhibition in vitro. Am. Rev. Respir. Dis. 116:65-72.

13. Gadek, J. E., G. A. Fells, and R. G. Crystal. 1979. Cigarette smoking induces functional antiprotease deficiency in the lower respiratory tract of humans. Science (Wash. DC). 206:1315-1316.

14. Cohen, A. B. 1979. The effects of in vivo and in vitro oxidative damage to purified $\alpha 1$-antitrypsin and to the enzyme-inhibiting activity of plasma. Am. Rev. Respir. Dis. 119:953-960.

15. Snider, G. L. 1981. The pathogenesis of emphysema: twenty years of progress. Am. Rev. Respir. Dis. 124:321-324.

16. Olsen, G. N., J. O. Harris, J. R. Castle, R. H. Waldman, and H. J. Karmgard. 1975. Alpha-1-antitrypsin content in the serum, alveolar macrophages, and alveolar lavage fluid of smoking and nonsmoking normal subjects. J. Clin. Invest. 55:427-430.

17. Hunninghake, G. W., J. E. Gadek, O. Kawanami, V. J. Ferrans, and R. G. Crystal. 1979. Inflammatory and immune processes in the human lung in health and disease: evaluation by bronchoalveolar lavage. Am. J. Pathol. 97:149-206.

18. Stone, P. J. 1983. The elastase-antielastase hypothesis of the pathogenesis of emphysema. Clin. Chest Med. 4:405-412.

19. Cohen, A. B., and H. L. James. 1982. Reduction of the elastase inhibitory capacity of alpha 1-antitrypsin by peroxides in cigarette smoke: an analysis of brands and filters. Am. Rev. Respir. Dis. 126:25-30.

20. Pryor, W. A., M. M. Dooley, and D. F. Church. 1986. The mechanisms of the inactivation of human alpha-1-proteinase inhibitor by gas-phase cigarette smoke. Adv. Free Radical Biol. Med. 2:161-188.

21. Carp, H., and A. Janoff. 1978. Possible mechanisms of emphysema in smokers: in vitro suppression of serum elastase-inhibitory capacity by fresh cigarette smoke and its prevention by antioxidants. Am. Rev. Respir. Dis. 118:617-621.

22. Fells, G., F. Ogushi, R. Hubbard, and R. G. Crystal. 1987. $\alpha 1$-Antitrypsin in the lower respiratory tract of cigarette smokers has a decreased association rate constant for neutrophil elastase. Am. Rev. Respir. Dis. 135:A291. (Abstr.)

23. Carp, H., F. Miller, J. R. Hoidal, and A. Janoff. 1982. Potential mechanism of emphysema: $\alpha 1$-proteinase inhibitor recovered from lungs of cigarette smokers contains oxidized methionine and has decreased elastase inhibitory capacity. Proc. Natl. Acad. Sci. USA. 79:2041-2045.

24. Abboud, R. T., T. Fera, A. Richter, M. Z. Tabona, and S. Johal. 1985. Acute effect of smoking on the functional activity of alpha 1protease inhibitor in bronchoalveolar lavage fluid. Am. Rev. Respir. Dis. 131:79-85.

25. Boudier, C., A. Pelletier, G. Pauli, and J. G. Bieth. 1983. The functional activity of $\alpha 1$-proteinase inhibitor in bronchoalveolar lavage fluids from healthy human smokers and non-smokers. Clin. Chim. Acta. 132:309-315.

26. Stone, P. J., J. D. Calore, S. E. McGowan, J. Bernardo, G. L. Snider, and C. Franzblau. 1983. Functional $\alpha 1$-protease inhibitor in the lower respiratory tract of cigarette smokers is not decreased. Science (Wash. DC). 221:1187-1189.
27. Fels, A. O. S., and Z. A. Cohn. 1986. The alveolar macrophage. J. Appl. Physiol. 60:353-369.

28. Nathan, C. F. 1987. Secretory products of macrophages. $J$. Clin. Invest. 79:319-326.

29. Hoidal, J. R., R. B. Fox, P. A. LeMarbe, R. Perri, and J. E. Repine. 1981. Altered oxidative metabolic responses in vitro of alveolar macrophages from asymptomatic cigarette smokers. Am. Rev. Respir. Dis. 123:85-89.

30. Hunninghake, G. W., and R. G. Crystal. 1983. Cigarette smoking and lung destruction: accumulation of neutrophils in the lungs of cigarette smokers. Am. Rev. Respir. Dis. 128:833-838.

31. Carp, H., and A. Janoff. 1980. Potential mediator of inflammation: phagocyte-derived oxidants suppress the elastase-inhibitory capacity of alpha 1-proteinase inhibitor in vitro. J. Clin. Invest. 66:987-995.

32. Hubbard, R., G. Fells, A. Cantin, F. Ogushi, M. Courtney, and R. Crystal. 1986. Recombinant DNA produced val ${ }^{358} \alpha 1$-antitrypsin more effectively resists oxidative inactivation by alveolar macrophages of cigarette smokers than plasma-derived M1M1 $\alpha 1$-antitrypsin or met $^{358} \alpha 1$-antitrypsin. Am. Rev. Respir. Dis. 133:A218.

33. Richter, A. M., R. T. Abboud, S. S. Johal, and T. A. Fera. 1986. Acute effect of smoking on superoxide production by pulmonary alveolar macrophages. Lung. 164:233-242.

34. Fulmer, J. D., W. C. Roberts, E. R. Von Gal, and R. G. Crystal. 1977. Small airways in idiopathic pulmonary fibrosis: comparison of morphologic and physiologic observations. J. Clin. Invest. 60:595610.

35. Saltini, C., A. J. Hance, V. J. Ferrans, F. Basset, P. B. Bitterman, and R. G. Crystal. 1984. Accurate quantification of cells recovered by bronchoalveolar lavage. Am. Rev. Respir. Dis. 130:650-658.

36. Rennard, S. I., G. Basset, D. Lecossier, K. M. O'Donnell, P. Pinkston, P. G. Martin, and R. G. Crystal. 1986. Estimation of volume of epithelial lining fluid recovered by lavage using urea as a marker of dilution. J. Appl. Physiol. 60:532-538.

37. Straus, S. D., G. A. Fells, M. D. Wewers, M. Courtney, L-H. Tessier, P. Tolstoshev, J.-P. LeCocq, and R. G. Crystal. 1985. Evaluation of recombinant DNA-directed E. coli produced $\alpha 1$-antitrypsin as an anti-neutrophil elastase for potential use as replacement therapy of $\alpha 1$-antitrypsin deficiency. Biochem. Biophys. Res. Commun. 130:1177-1184.

38. Johnston, R. B. 1981 . Secretion of superoxide anion. In Methods for Studying Mononuclear Phagocytes. D. O. Adams, R. J. Edelson, and H. S. Koven, editors. Academic Press, New York. 489-497.

39. Nathan, C. F. 1981. Release of hydrogen peroxide. In Methods for Studying Mononuclear Phagocytes. D. O. Adams, R. J. Edelson, and H. S. Koven, editors. Academic Press, New York. 499-510.

40. Cox, W., A. M. Johnson, M. K. Fagerhol. 1980. Report of nomenclature meeting for $\alpha 1$-antitrypsin. Hum. Genet. 53:429-433.

41. Nukiwa, T., K. Satoh, M. L. Brantly, F. Ogushi, G. A. Fells, M. Courtney, and R. G. Crystal. 1986. Identification of a second mutation in the protein coding sequence of the Z-type alpha 1-antitrypsin gene. J. Biol. Chem. 261:15989-15994.

42. Sugiura, M., S. Hayakawa, T. Adachi, Y. Ito, K. Hirano, and S. Sawaki. 1981. A simple one-step purification of human $\alpha 1$-proteinase inhibitor by immunoadsorbent column chromatography. J. Biochem. Biophys. Methods. 5:243-249.

43. Courtney, M., A. Buchwalder, L.-H. Tessier, M. Jaye, A. Benavente, A. Balland, V. Kohli, R. Lathe, P. Tolstoshev, and J.-P. LeCocq. 1984. High-level production of biologically active human $\alpha 1$-antitrypsin in Escherichia coli. Proc. Natl. Acad. Sci. USA. 61:669-673.

44. Courtney, M., S. Jallat, L.-H. Tessier, A. Benavente, R. G. Crystal, and J.-P. LeCocq. 1985. Synthesis in E. coli of $\alpha 1$-antitrypsin variants of therapeutic potential for emphysema and thrombosis. $\mathrm{Na}$ ture (Lond). 313:149-151.

45. George, P. M., J. Travis, M. C. M. Vissers, C. C. Winterbourn, 
and R. W. Carrell. 1984. A genetically engineered mutant of $\alpha 1$-antitrypsin protects connective tissue from neutrophil damage and may be useful in lung disease. Lancet. ii:1426-1428.

46. Travis, J., M. Owen, P. George, R. Carrell, S. Rosenberg, R. A. Hallewell, and P. J. Barr. 1985. Isolation and properties of recombinant DNA produced variants of human $\alpha 1$-proteinase inhibitor. $J$. Biol. Chem. 260:4384-4389.

47. Rosenberg, S., P. J. Barr, R. C. Najarian, and R. A. Hallewell. 1984. Synthesis in yeast of a functional oxidation-resistant mutant of human $\alpha$ 1-antitrypsin. Nature (Lond.). 312:77-80.

48. Janoff, A., C. George-Nascimento, and S. Rosenberg. 1986. A genetically engineered, mutant human alpha-1-proteinase inhibitor is more resistant than the normal inhibitor to oxidative inactivation by chemicals, enzymes, cells, and cigarette smoke. Am. Rev. Respir. Dis. 133:353-356.

49. Bieth, J. C. 1984. In vivo significance of kinetic constants of protein proteinase inhibitors. Biochem. Med. 32:387-394.
50. Niewoehner, D. E., J. Kleinerman, and D. B. Rice. 1974 Pathologic changes in the peripheral airways of young cigarette smokers. N. Engl. J. Med. 291:755-758.

51. Campbell, E. J., and M. S. Wald. 1983. Fate of human neutrophil elastase following receptor-mediated endocytosis by human alveolar macrophages. J. Lab. Clin. Med. 101:527-536.

52. McGowan, S. E., R. D. Arbeit, P. J. Stone, and G. L. Snider. 1983. A comparison of the binding and fate of internalized neutrophil elastase in human monocytes and alveolar macrophages. Am. Rev. Respir. Dis. 128:688-694.

53. van oud Alblas, A. B., and R. van Furth. 1979. Origin, kinetics, and characteristics of pulmonary macrophages in the normal steady state. J. Exp. Med. 149:1504-1518.

54. Bitterman, P. B., L. E. Saltzman, S. Adelberg, V. J. Ferrans, and R. G. Crystal. 1984. Alveolar macrophage replication: one mechanism for the expansion of the mononuclear phagocyte population in the chronically inflamed lung. J. Clin. Invest. 74:460-469. 